\title{
ILCEA
}

Revue de l'Institut des langues et cultures

d'Europe, Amérique, Afrique, Asie et Australie

15 | 2012

Les mots de la crise

« Acte de Dieu » ou « Erreur humaine »? - Analyse argumentative du débat relative à la crise de l'automobile de Détroit (2008)

"Act of God" or "Human error"? - Argumentative analysis of the debate surrounding the 2008 Detroit automobile crisis

\section{Rudi Palmieri}

\section{OpenEdition}

\section{Journals}

Édition électronique

URL : http://journals.openedition.org/ilcea/1131

DOI : 10.4000/ilcea. 1131

ISSN : 2101-0609

\section{Éditeur}

UGA Éditions/Université Grenoble Alpes

Édition imprimée

ISBN : 978-2-84310-220-2

ISSN : $1639-6073$

\section{Référence électronique}

Rudi Palmieri, « «Acte de Dieu » ou «Erreur humaine »? - Analyse argumentative du débat relative à la crise de l'automobile de Détroit (2008) », ILCEA [En ligne], 15 | 2012, mis en ligne le 30 janvier 2012 consulté le 01 mai 2019. URL : http://journals.openedition.org/ilcea/1131 ; DOI : 10.4000/ilcea.1131

Ce document a été généré automatiquement le 1 mai 2019.

(c) ILCEA 


\title{
«Acte de Dieu » ou « Erreur
} humaine »? - Analyse argumentative du débat relative à la crise de l'automobile de Détroit (2008)

\author{
"Act of God" or "Human error"? - Argumentative analysis of the debate \\ surrounding the 2008 Detroit automobile crisis
}

Rudi Palmieri

\section{NOTE DE L'AUTEUR}

L'auteur exprime ici sa gratitude envers les deux relecteurs anonymes de ce travail et tient à les remercier pour leurs observations et suggestions très constructives.

\section{Introduction : l'argumentation dans un contexte de crise financière}

Cet article analyse le discours généré autour de la crise de l'industrie automobile américaine en 2008 selon une approche relevant des théories de l'argumentation. Celle-ci s'avère particulièrement intéressante par rapport à des situations de crise économique qui permettent d'étudier les stratégies communicatives mises en œuvre par les entreprises pour récupérer ou préserver la confiance de leurs groupes d'intérêt ( stakeholders) principaux (Benoit, 1997 ; Palmieri, 2009). L'approche discursive de la crise s'intéresse plus précisément aux phénomènes lexicaux et interlinguistiques (comme l'introduction de termes nouveaux, leur traduction et diffusion). Les aspects 
argumentatifs ne doivent pas être sous-estimés puisque les décisions stratégiques à prendre pendant une crise et les débats qui accompagnent les mesures débattues sont souvent caractérisés par un échange d'arguments où des intérêts, des opinions et des préoccupations différents sont confrontés.

2 L'argumentation comporte une activité de traduction lato sensu, car les raisons qui portent un sujet à soutenir une thèse peuvent conduire à l'acceptation de cette thèse par l'interlocuteur seulement si ces raisons lui sont communiquées d'une manière claire et efficace qui lui permet de les comprendre et de les faire siennes. À cet égard, l'argumentation ne peut pas être proprement étudiée en négligeant son contexte (Rigotti et Greco Morasso, 2009b) parce qu'elle ne correspond pas seulement à une procédure logique, mais à une forme d'interaction communicative raisonnable dans laquelle on cherche à convaincre l'interlocuteur d'une thèse en lui donnant les raisons de l'accepter (van Eemeren et Grootendorst, 2004 ; Rigotti et Greco Morasso, 2009a).

La théorie de l'argumentation distingue, d'une part, des contextes communicatifs où le but de l'interaction argumentative est le savoir, la certitude au sujet d'une proposition douteuse et, d'autre part, des situations dont le but est une décision prise par l'interlocuteur, qui est en fait le décideur (van Eemeren, 2010 ; van Rees et Rigotti, 2011). Dans le domaine de la finance, les différentes interactions communicatives activées sont orientées en général vers la prise de décision: elles se caractérisent donc, au niveau argumentatif, par des raisonnements de nature pragmatique visant le bien-fondé d'une bonne décision (Palmieri et Palmieri, 2011). Même si les décisions financières impliquent des connaissances très techniques et spécialisées, l'argumentation financière doit se développer autour du décideur et tenir compte de ses compétences spécifiques, en «traduisant» des données complexes en un chemin argumentatif que le décideur doit être capable de parcourir.

4 Dans une situation particulière telle qu'une crise, ce fait devient évident parce que le monde de la finance envahit celui de l'économie réelle et de la société en général et la communication financière doit donc s'adapter à différentes catégories d'interlocuteurs : les banques doivent persuader leurs petits comme leurs gros clients de continuer à leur faire confiance; les entreprises peuvent être obligées de solliciter l'aide publique de l'État, en demandant donc aux gouvernements de leur céder l'argent des citoyens ; l'État lui-même peut se trouver dans la situation où il doit obtenir l'appui d'autres pays.

5 Dans ce cadre, la demande de soutien public est particulièrement intéressante parce qu'il s'agit ici d'une action financière proposée à un «investisseur » qui n'a ni une mission, c'est-à-dire une raison d'être financière, ni des intentions spéculatives. Il faut démontrer aux institutions publiques et, donc, à l'opinion publique, que l'entreprise mérite de recevoir ces fonds qui ne pourront pas, par conséquent, être investis dans d'autres services publics.

Dans cet article nous nous proposons de présenter une analyse argumentative des passages les plus significatifs qui ont caractérisé le débat sur l'aide publique aux trois grands constructeurs automobiles de Détroit, c'est-à-dire General Motors, Chrysler et Ford. Ce débat a accompagné la première phase de la crise financière, juste après la faillite de la banque d'affaires Lehman Brothers en septembre 2008. Il s'agit donc d'une crise dans la crise, bien que les entreprises aient soutenu l'idée d'un lien de causalité entre leurs difficultés et le resserrement du crédit. En revanche, plusieurs critiques ont été formulées à l'encontre des trois constructeurs automobiles, accusés de n'avoir pas été 
capables, indépendamment de la crise financière, de s'adapter aux développements du secteur. La différence principale entre les deux points de vue concerne les implications sur la responsabilité de la direction de ces entreprises : pour les uns, les dirigeants ne sont pas coupables et s'il y a des responsables, ils ne se trouvent pas dans les entreprises; pour les autres, les dirigeants portent une responsabilité directe évidente. Donc, pour les uns, la crise du secteur est un événement provoqué par la crise financière alors que pour les autres, la crise des trois entreprises est le résultat d'actions et décisions humaines.

On doit souligner que l'ambiguïté « événement-action » est très fréquente dans le monde économique, souvent décrit comme s'il s'agissait d'un secteur de la réalité physique, immuable et gouvernée par le déterminisme, plutôt que le produit d'actions humaines plus ou moins libres et conscientes. L'exemple suivant, concernant un événement des premiers mois de la crise, est significatif. C'est une déclaration faite par Marcel Ospel, dirigeant d'UBS, devant ses actionnaires en février 2008, qui comprend un cadrage ( framing) métaphorique d'une grande importance stratégique (Rocci, 2009) :

We were therefore all the more disappointed that we had failed to recognize the signals from the US housing market in time - early enough to allow us to take appropriate action that would have kept UBS out of the storm that then broke over the financial markets. We were not, of course, the only bank to be affected by these events. (Mon italique)

La crise est vue ici presque comme un phénomène naturel (un événement), dont l'homme peut seulement reconnaître les signaux pour chercher à prévenir ses effets négatifs et à se protéger avant que la catastrophe n'ait lieu ${ }^{1}$. La crise est décrite comme une tempête qui s'est abattue sur les marchés financiers, dont UBS et d'autres institutions font partie.

Décrire la réalité économique comme un ensemble de phénomènes naturels ne constitue pas une exception et le débat sur la crise de Détroit n'en fait pas exception:

Two of Detroit's Big Three auto makers are on the verge of collapse, industry executives told a Senate panel Tuesday, urging emergency assistance from the government to avert what they said would be an economic catastrophe. (The Wall Street Journal Europe, 11/11/2008)

Est-ce qu'une crise doit être vue comme une catastrophe naturelle ou comme une faillite due à des erreurs humaines? Dans le débat concernant l'aide publique à Détroit cette différence a joué un très grand rôle.

11 Après avoir fourni une explication de la méthode d'analyse, nous résumerons brièvement les principales étapes historiques de l'affaire Détroit. Nous présenterons une analyse des raisons principales avancées par les directeurs des trois entreprises en vue de convaincre le gouvernement d'augmenter le montant des fonds alloués; nous nous pencherons ensuite sur les nombreuses critiques formulées à l'encontre des Big Three, en analysant la contre-argumentation, en particulier les raisons avancées par le journaliste Paul Ingrassia, responsable du bureau du Wall Street Journal (WSJ) à Détroit et lauréat du Prix Pulitzer en 1993 pour ses articles sur General Motors.

\section{Méthode d'analyse argumentative du débat}

12 Afin d'analyser un débat en partant d'une perspective argumentative, on doit d'abord identifier la question qui se pose (issue), les parties et leur position, qui correspond en général à une thèse, mais qui peut aussi se limiter à soulever des doutes qui déclenchent 
une attitude argumentative. Finalement, les arguments avancés pour soutenir la ou les thèses sont identifiés et reconstruits dans leur configuration inférentielle.

En ce qui concerne la question (issue), on peut distinguer différents niveaux qui correspondent à différents stock issues ${ }^{2}$, dans la doctrine éponyme (fondée sur les status causae de la rhétorique ancienne) utilisée pour l'étude des débats relatifs à l'introduction d'une nouvelle mesure politique. La discussion porte sur la nécessité d'intervenir dans un contexte pour le changer ( «need for change» issue) et examine si cette nécessité est occasionnée par la situation présente («blame » issue), si l'intervention en question peut effectuer le changement visé («cure » issue) et si la réalisation de la mesure ne comporte pas d'effets secondaires (« cost » issue).

14 L'analyse strictement argumentative que nous proposons est fondée sur l'approche pragma-dialectique (van Eemeren et Grootendorst, 2004) pour ce qui concerne la vue d'ensemble analytique, c'est-à-dire, la reconstruction de la thèse et de la structure argumentative qui la soutient. Pour l'analyse inférentielle, nous adoptons l'Argumentum Model of Topics (AMT), élaboré par le groupe de recherche de Lugano (Rigotti, 2006, 2009; Rigotti et Greco Morasso, 2009, 2010 ; Rigotti et Palmieri, 2010). L'analyse porte sur des articles d'opinion publiés dans plusieurs médias (comme le Wall Street Journal, BBC News) entre la fin de 2008 et le début de 2009 et sur d'autres déclarations faites par les dirigeants des Big Three au cours de la même période.

\section{Le cas Détroit}

\section{Reconstruction chronologique}

Avant de livrer une reconstruction chronologique de ce cas, il faut tout d'abord préciser que celle-ci peut elle-même être orientée par un point de vue déjà existant sur la question. Les implications sont en effet très différentes selon que l'on choisit de commencer par ce qui s'est passé dans l'industrie automobile après le début de la crise en 2007 ou que l'on considère les problèmes vécus par les Big Three plusieurs années en amont. Comme Cicéron avait déjà remarqué dans Rhetorica ad Herennium (Cancelli, éd. 1992), la reconstruction des faits (narratio) n'est jamais neutre, et souvent fortement orientée en fonction de la cause soutenue par le rhéteur.

Donc nous partirons simplement de la décision institutionnelle en question, à savoir, celle que devait prendre l'État américain de soutenir ou non les trois entreprises. Les différentes narrations (au sens de narratio) seront exposées à partir des différentes stratégies argumentatives mises en œuvre par les acteurs impliqués.

Immédiatement après la chute de la banque Lehman Brothers en septembre 2008, les trois entreprises sollicitent un soutien financier au gouvernement américain qui avait créé le fonds TARP (Troubled Assets Recovery Plan) pour venir en aide à l'économie. Le 19 novembre 2008, une session spéciale du Congrès convoque les trois dirigeants, Rick Wagoner (GM), Robert Nardelli (Chrysler), et Alan Mulally (Ford), pour entendre leurs arguments en faveur d'un tel soutien. La décision est renvoyée au mois de décembre, moment où le Président George Bush, peu avant de quitter la Maison Blanche, décide de satisfaire partiellement cette requête en octroyant un prêt de 13,4 milliards de dollars aux trois entreprises. Avec le nouveau gouvernement du président Barack Obama, GM et Chrysler reçoivent des sommes supplémentaires, mais à des conditions plus strictes: le 
directeur général de GM doit renoncer à son poste ; Chrysler ne recevra les fonds qu'après avoir signé un accord préalable avec Fiat au terme duquel l'entreprise italienne s'engagerait à prendre une part élevée du capital de Chrysler.

Malheureusement, le soutien obtenu ne permettra pas à GM de survivre et, au mois de juin suivant, cette entreprise, qui compte parmi les plus importantes des États-Unis, doit se déclarer en faillite.

\section{La question principale et les parties argumentatives}

19 L'analyse qui suit se focalise sur la période de la session spéciale du Congrès, en novembre 2008. Avant cet événement, le débat, très acharné, tourne autour d'une question principale que l'on peut résumer ainsi : le gouvernement des États-Unis doit-il soutenir les trois entreprises de Détroit?

D'une part, il y a les trois entreprises représentées par leurs dirigeants qui, ayant sollicité le soutien de l'État, défendent nécessairement la thèse affirmative : «le gouvernement doit financer les trois entreprises ». D'autre part, il y a plusieurs adversaires qui tiennent la thèse contraire («le gouvernement ne doit pas...») ou une thèse partiellement différente (« le gouvernement devrait les soutenir mais à condition que...»).

21 La formulation de la question que nous avons proposée indique clairement la nature pragmatique de cette décision. Le soutien éventuel du gouvernement relève d'une politique financière qui relève à son tour de la doctrine des stock issues. En effet, la décision de mettre en œuvre certaines mesures politiques dépend de différentes questions subordonnées (sub-issues) : l'espoir de résoudre effectivement le problème en question; l'importance relative des mesures alternatives qui ne pourraient pas être activées; le mérite de ceux qui auraient bénéficié de cette mesure, etc. Ici, le besoin de changement ne semble pas être mis en discussion. Le resserrement du crédit rend l'aide publique nécessaire pour soutenir l'économie nationale là où la crise ne permet plus de continuer l'activité d'une entreprise. En revanche, les questions de blame issue et cure issue sont ouvertes à la discussion. Dans la première on essaie de déterminer si les problèmes spécifiques de Détroit ont été occasionnés par cette crise financière contre laquelle le plan de financement (TARP) a été conçu. Ici, nous pouvons identifier une nette différence d'opinions. Il en va de même pour le débat de cure issue, dans lequel on se demande si l'aide aux Big Three permettrait vraiment aux entreprises de résoudre leurs problèmes.

\section{Les arguments présentés par The Big Three : la crise comme « acte divin »}

Bien qu'il y ait des différences entre les argumentations des trois entreprises, elles peuvent être résumées par l'extrait suivant, tiré d'un article du WSJ en date du 11 novembre 2008: "The executives defended the management of their companies, saying the need for emergency government support stems from the economic crisis, not poor decision-making ».

Cette citation permet de dégager deux constats importants : (1) la discussion concernant la question du financement des Big Three par le gouvernement se transforme en un débat sur la responsabilité des entreprises elles-mêmes, donc la blame issue; (2) les trois 
entreprises se déclarent non responsables de la situation qui les a amenées à demander ce soutien, alors que leurs adversaires soutiennent le contraire.

Dans les annexes, le lecteur trouvera un article d'opinion écrit par Rick Wagoner, directeur général de GM, et publié dans le WSJ le jour de la session du Congrès. Le titre laisse entendre d'entrée de jeu la nature argumentative du texte, car il annonce que l'article exposera les raisons qui justifieraient l'aide du gouvernement à GM («deserves support »). Plus précisément, avec l'expression « pourquoi GM mérite un soutien » (« why GM deserves support »), Wagoner présente sa thèse et assume la charge de la preuve (burden of proof dans la théorie de l'argumentation), c'est-à-dire l'engagement à donner des raisons qui justifient la thèse. De plus, le sous-titre contient une argumentation plus complexe : «le soutien à court terme par le gouvernement peut préserver un secteur industriel vital». Il est dès lors possible de reconstruire la micro-argumentation suivante :

- Question : Est-ce que GM est digne de recevoir le soutien du Gouvernement ?

- Thèse de R. Wagoner : GM est digne de recevoir le soutien du Gouvernement.

- Argument : Le soutien à court terme par le gouvernement peut préserver un secteur industriel vital.

Il convient ici de souligner la caractérisation du bailout (sauvetage) comme «soutien à court terme ", en particulier en considérant le début de l'article (lignes 1-4). Wagoner rappelle la question en jeu en précisant que le soutien demandé concerne la période " extraordinaire " de la crise financière (" whether or not the government should assist the industry during this extraordinary turmoil »). C'est ainsi que la situation oriente l'interprétation vers la thèse selon laquelle la demande est due à l'impact de la crise financière sur l'industrie. Donc, l'origine des problèmes subis par GM est à rechercher à l'extérieur de l'entreprise. Comme on verra plus loin, selon cette argumentation la crise de l'industrie automobile est représentée comme une sorte d'acte divin, c'est-à-dire un événement dont l'origine ne peut pas être attribuée à la responsabilité de ceux qui vont en souffrir. Dans la tradition anglo-saxonne, la notion juridique de «Act of God » se réfère à un événement que l'être humain ne peut pas contrôler. Cette notion joue un rôle central dans le domaine de l'assurance, car elle peut exonérer d'une responsabilité la personne qui aurait dû l'assumer.

Dans les articles analysés, cette manœuvre argumentative est présente dans plusieurs passages (voir exemples en annexe 3). Par exemple, le dirigeant de Ford déclare que la pression sur leur secteur est le résultat de la crise financière, laquelle n'a donc pas été le résultat de leur gestion :

This unprecedented pressure on our industry, which is the result of a financial crisis that was not of our industry's making, is coming just at the time when our efforts to restructure Ford have finally begun to bear fruit. (Mon italique)

Dans ce passage, la thèse implicite peut être reconstruite de la façon suivante «nous ne sommes pas responsables des difficultés de notre industrie ». L'argumentation en faveur de la thèse unit le fait - Datum dans la théorie de l'argumentation (Toulmin, 1958) - que « la crise financière n'a pas été la conséquence d'une action des entreprises automobiles » avec un point de départ apparemment partagé affirmant que «c'est la crise financière qui a poussé l'industrie automobile au bord de la faillite». Cette dernière prémisse est proprement nommée Endoxon (Rigotti et Greco Morasso, 2010), une notion introduite par Aristote pour se référer aux opinions, aux valeurs et aux principes partagés dans une 
communauté, une culture, un contexte social et qui, par conséquent, sont souvent présupposés dans un discours, laissés implicites ou simplement suggérés.

Figure 1. - Structure inférentielle de l'argument contre la responsabilité de Détroit.

\section{Endoxon : C'est la crise financière qui a conduit l'industrie automobile au bord de la faillite.}

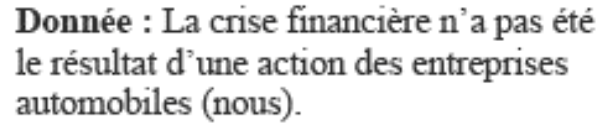

Donnée : La crise financière n'a pas été le résultat d'une action des entreprises automobiles (nous).

Comme la structure en Y (Rigotti et Greco Morasso, 2010) dans la figure 1 le montre, l' Endoxon et le Datum justifient la thèse (conclusion finale) grâce à une maxime, c'est-à-dire une connexion inférentielle qui dérive d'un domaine ontologique, le lieu (lat. locus; gr. topos). Le lieu joué ici est celui de la cause finale (Rigotti, 2008), c'est-à-dire la relation entre l'agent, l'action et le but (figure 2).

Plusieurs maximes appartiennent au même lieu argumentatif. Dans ce cas, la maxime est la suivante : « Si un agent n'est pas à l'origine d'un résultat qui a engendré une situation négative, il ne peut être considéré comme responsable de cette situation.» La responsabilité, en effet, dérive de l'activation libre et consciente (donc d'une vraie décision) d'une chaîne causale qui, de manière prévisible, conduira au résultat qui correspond au but recherché par l'agent.

La maxime active une procédure logique (modus ponens dans ce cas) qui conduit à la conclusion finale (la thèse). La prémisse mineure de ce syllogisme hypothétique correspond à la conclusion d'un syllogisme catégoriel combinant l'Endoxon et le Datum. Donc, cette prémisse mineure représente le point de rencontre entre la dimension logicoinférentielle de l'argumentation et la dimension matérielle, contextuelle, et donc concrète de l'interaction argumentative. 
Figure 2. - La structure de l'action (Rigotti, 2008).

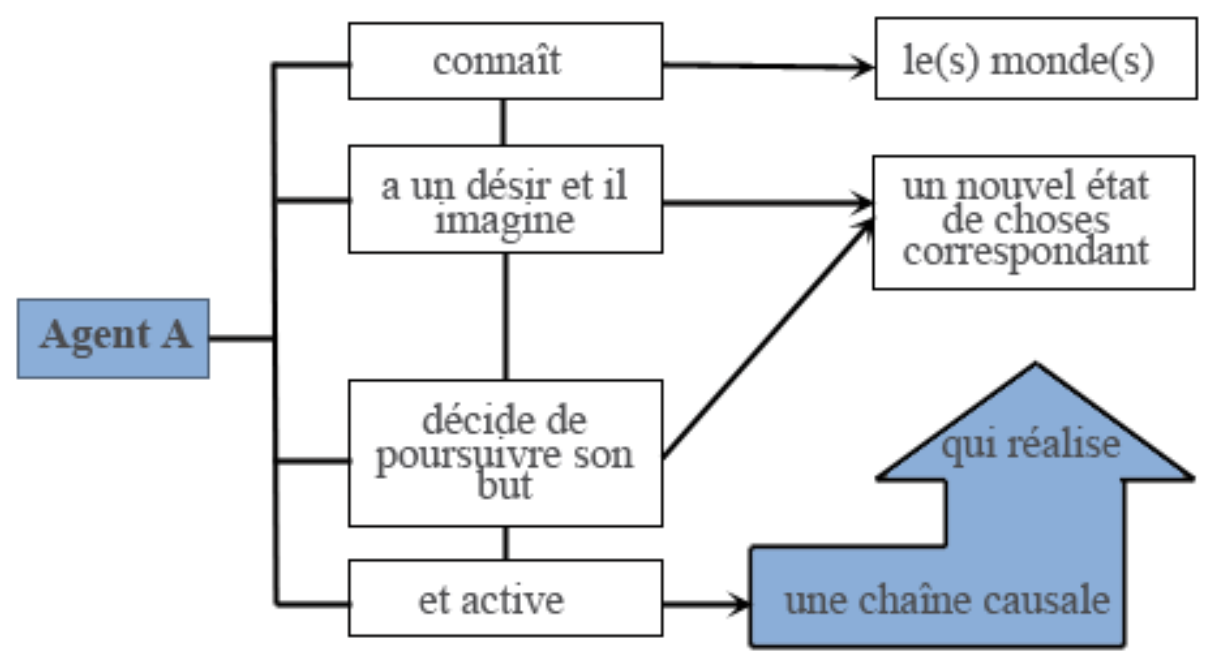

Si nous revenons à l'article publié par Wagoner, on voit que dans le premier paragraphe, il semble déclarer la question encore ouverte car insuffisamment débattue. En général, une question doit être suffisamment pertinente et importante pour être digne d'argumentation, c'est-à-dire pour retenir l'intérêt de l'interlocuteur et le convaincre d'assumer une attitude argumentative. Dans ce cas, Wagoner observe que, même si le débat a déjà fait l'objet d'une longue discussion (« much has been said about... »), il doit être poursuivi parce que, à son avis, il n'a pas été tenu compte de certains éléments importants qui, une fois pris en considération d'un point de vue argumentatif, auraient influencé la décision finale.

En se référant à "many critics", Wagoner invoque ceux qui sont évidemment en désaccord avec son point de vue et fait référence à un argument brandi par ses adversaires: GM et les autres firmes automobiles n'avaient pas entrepris les modifications nécessaires pour mériter le soutien :

- Question : Est-ce que GM est digne de recevoir le soutien du Gouvernement?

- Thèse des adversaires : GM n'est pas digne de recevoir le soutien du Gouvernement.

- Argument : GM n'a pas réalisé les changements nécessaires.

Le texte de Wagoner peut être découpé en deux grandes parties qui correspondent à deux arguments en faveur de la thèse défendue par GM. Dans la première partie (lignes 8-25) le contre-argument que nous venons de mentionner est réfuté en indiquant les nombreuses réductions de coûts et l'innovation dans la production de nouvelles voitures, deux revendications qui constituent un argument composé qui démontrerait que les mesures prises permettent à GM de satisfaire à la condition sine qua non d'obtention de l'aide publique. Avec cette argumentation, l'absence d'erreur humaine, et donc l'interprétation de la crise comme « acte divin », est donc déjà implicite. 
Figure 3. - R. Vue d'ensemble analytique de la discussion entre GM et ses adversaires.

QUESTION : Est-ce que GM est digne de recevoir le soutien du Gouvermement?

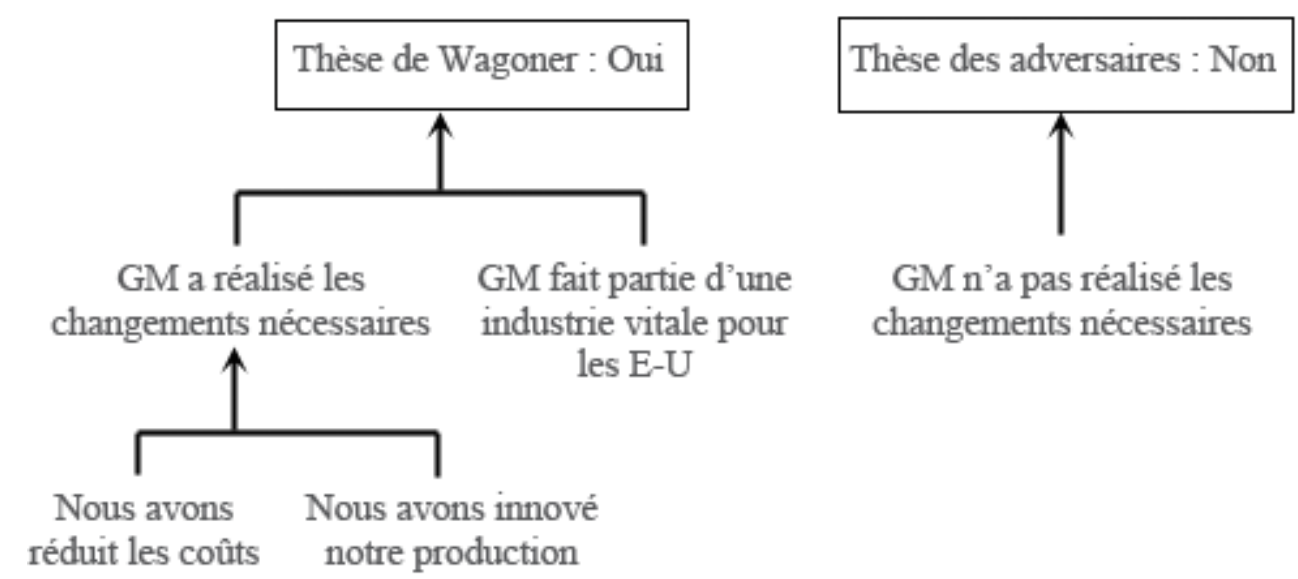

Comme la vue d'ensemble analytique le montre (figure 3), GM serait digne du soutien public sur la base d'un autre motif également qui est exposé dans la deuxième partie du texte (lignes 26-47): "GM's commitment to these new products and technologies will help everyone »- «Everyone » étant les nombreux et divers «stakeholders » (parties prenantes) impliqués, comme l'élucide le début de l'intervention (testimony) de Wagoner devant le Congrès :

Good afternoon, Mr. Chairman.

I'm Rick Wagoner, Chairman and Chief Executive Officer of General Motors. Thank you for the opportunity to speak today about the future of America's domestic auto industry.

I'd like to acknowledge for the Committee the audiences that I represent:

General Motors directly employs approximately 96,000 people in the United States. We have 6,500 dealers across the country, who employ another 340,000 .

Last year, we purchased more than $\$ 30$ billion of goods and services from more than 2,000 suppliers in 46 states.

Our pension program covers nearly 475,000 retirees and spouses, and our health benefits extend to about one million Americans.

We have about one million registered stockholders.

And 70 million of our vehicles are registered to U.S. citizens... 22 million of them purchased in the last 5 years.

(18 novembre 2008, source: <http://s.wsj.net/public/resources/documents/ WSJ_WagonerTestimony-081118.pdf>)

On observe que le sujet du débat n'est pas présenté comme une situation problématique pour GM, mais comme la clé du futur de l'industrie automobile américaine. Ensuite, la liste de ceux qui sont liés au sort de GM est détaillée comme preuve que les personnes qui bénéficieraient d'une aide à GM ou, inversement, qui souffriraient de la faillite de la société, appartiennent à plusieurs catégories d'acteurs sociaux impliqués : employeurs, concessionnaires, fournisseurs, retraités, investisseurs, actionnaires et clients. 

interlocuteurs-décideurs que l'État représente et au nom desquels il doit agir. La décision du gouvernement doit ainsi être argumentée en fonction des enjeux de ces parties prenantes. Comme soutenir GM signifie soutenir les citoyens, le refus de financer les entreprises automobiles signifierait, selon la logique de Wagoner, que le gouvernement a manqué à son mandat envers les citoyens et a ainsi, failli à sa mission. La manœuvre de Wagoner devient ainsi une sorte de menace ( ${ }^{3}$ si vous ne nous aidez pas, vous n'êtes pas un bon gouvernement et ne méritez pas de rester au pouvoir »).

L'article de Wagoner conclut sur l'argument anticipé dans le titre, en spécifiant que le soutien à court terme servira de pont pour traverser la crise financière («bridge the current financial crisis »). Avec cette formulation, Wagoner semble vouloir dire que le cours de GM était à la hausse mais que, tout à coup, une tempête (la crise) a créé un fossé l'empêchant de continuer son ascension. Cette représentation présuppose que (1) avant la crise financière, GM était une entreprise couronnée de succès ; (2) après avoir reçu l'aide du gouvernement, GM renouera avec le succès.

\section{La critique de l'analogie « crise-catastrophe »}

Le désaccord total avec cette représentation, exprimé par de nombreux commentateurs, est bien illustré dans les passages suivants :

This situation doesn't stem from the recent meltdown in banking and the markets. GM, Ford and Chrysler have been losing billions since 2005, when the U.S. economy was still healthy. (Ingrassia, WSJ, 25 octobre 2008)

In all this lies a tale of hubris, missed opportunities, disastrous decisions and flawed leadership of almost biblical proportions. In fact, for the last 30 years Detroit has gone astray, repented, gone astray and repented again in a cycle not unlike the Israelites in the Book of Exodus (ibid.)

This is Larry Sinclair. He lives in California and drives a Honda (HMC), and he doesn't want Detroit to have a bailout. Here's what he wrote to his congressman : "They have been asleep for 30 years and have made their own problems. So why should I help bail them out?" (interview de R. Nardelli, Business Week, 18 novembre 2008)

But you know what the critics are saying. [...] They're saying basically you failed to lead, and now you have your hand out saying, "Help us". (interview de A. Mulally, $\mathrm{CNN}, 18$ novembre 2008)

Parmi les nombreux articles de presse qui ont pris parti contre le bailout ${ }^{4}$, nous en retiendrons un qui est, selon nous, particulièrement frappant (voir annexe 2). Il porte sur un texte écrit par Paul Ingrassia et publié dans le WSJ le 21 novembre 2008 sous le titre significatif: "The Auto Makers Are Already Bankrupt». Une grande partie de cet article (lignes 7-51) est consacrée à la destruction de cinq mythes, c'est-à-dire de cinq Endoxa, présupposés dans les argumentations en faveur du soutien public à Detroit: la faillite n'est pas une option, changer les dirigeants serait inutile, faillite signifie mort, utiliser l'argent public pour produire de meilleures voitures sauverait Detroit, une fusion entre GM et Chrysler bénéficierait aux deux.

Cependant, à nos yeux, le passage le plus intéressant dans ce texte se trouve au début (lignes 1-4). En quatre lignes, Ingrassia développe un discours clairement polyphonique (Ducrot, 1984), car, avant d'exposer sa position, il mentionne une argumentation contraire à la sienne, qui justifierait le bailout. Cette argumentation se fonde sur une 
analogie entre la crise du secteur automobile et l'ouragan Katrina, qui en 2005 avait touché fortement la Nouvelle-Orléans :

- Thèse : Le soutien du gouvernement se justifie pour la crise de Détroit.

- Argument : Dans le cas de l'ouragan Katrina, le fait d'être un « acte divin » a justifié le soutien du gouvernement. dans la figure 4. L'argument se fonde visiblement sur l'Endoxon selon lequel la crise de Détroit est un « Acte de Dieu » (car elle est occasionnée par la crise financière).

Figure 4. - Structure inférentielle de l'argument en faveur du soutien aux Big Three.

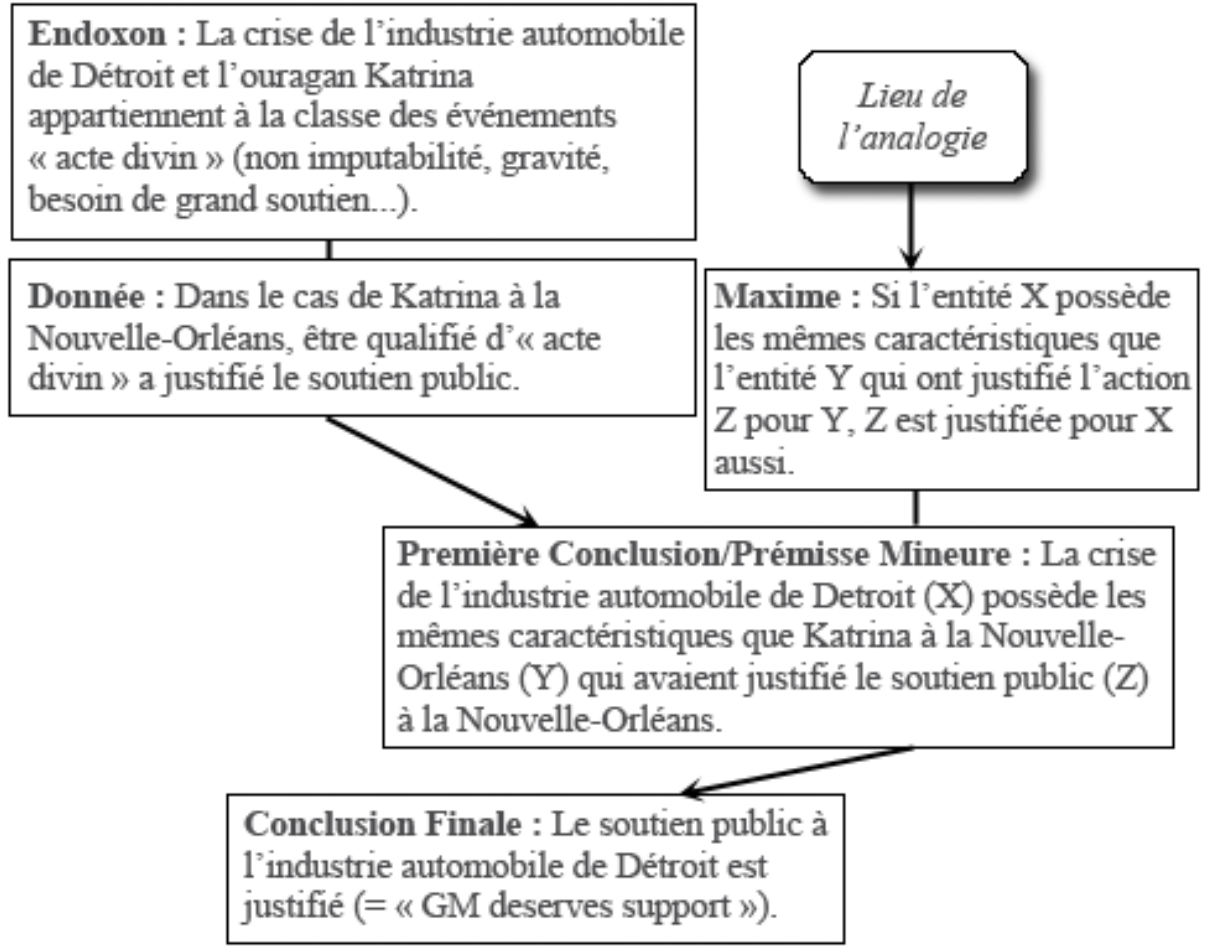

Ingrassia réfute cette comparaison en rappelant que Katrina est un phénomène naturel, tandis que la crise de Detroit est le résultat d'une action humaine, donc un état d'affaires occasionné pas des sujets responsables. Ingrassia essaie de démasquer une manipulation qu'il qualifie de "victimisation ", amenant à considérer une faillite humaine comme une catastrophe naturelle et, par conséquent, les coupables comme victimes :

- Thèse de P. Ingrassia : Le soutien public n'est pas justifié pour la crise de l'industrie automobile de Détroit.

- Argument : Katrina est un Acte de Dieu, la crise de l'industrie automobile de Détroit est un acte humain.

La figure 5 montre la configuration inférentielle de cette contre-argumentation : 
Figure 5. - Structure inférentielle de la contre-argumentation de Paul Ingrassia.

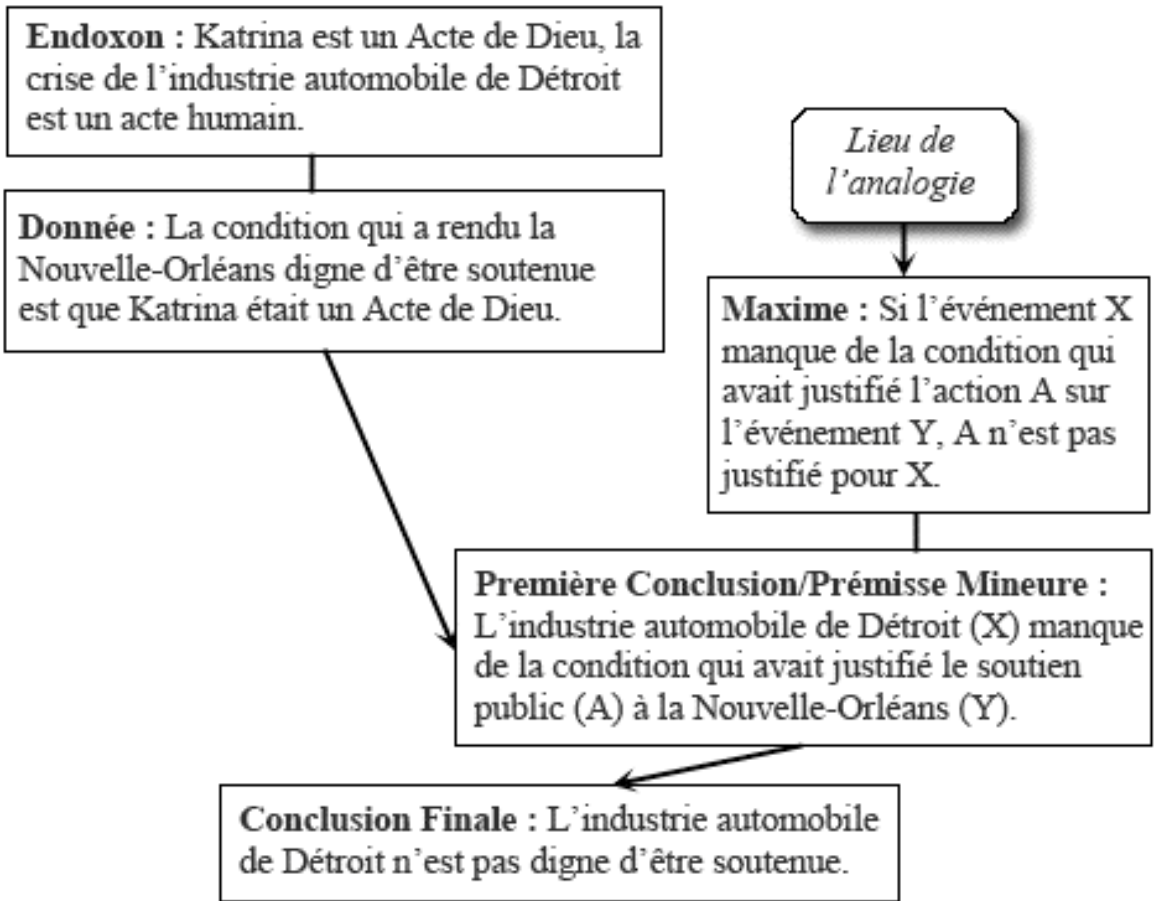

\section{Conclusion}

Cet article a identifié et analysé les argumentations principales qui ont caractérisé le débat sur le soutien public aux Big Three de Détroit. Nous avons tenté de démontrer que le fait d'interpréter un phénomène économique comme événement ou comme action peut avoir des implications très importantes pour les décisions en jeu. Ces interprétations sont exploitées dans les argumentations en faveur ou contre certaines mesures. Pour les dirigeants, la crise du secteur automobile est vue comme un événement provoqué par la crise financière qui a compromis la possibilité, pour les entreprises, de continuer leur activité, ce qui présuppose une absence de responsabilité. Pour plusieurs critiques, la crise de Détroit précède la crise générale, et elle est due également à des erreurs de gestion. Dans ce cadre, les dirigeants sont partiellement responsables des problèmes affectant les entreprises.

Établir la responsabilité - ou non - d'une personne est une entreprise complexe. En effet, bien que la crise financière, comme celle de Détroit, soit pour certains le résultat de mauvaises décisions, pour d'autres, comme pour beaucoup d'employés de banque, ces résultats constituent des événements et donc, relève d'un Acte de Dieu.

À travers cette étude, nous espérons avoir démontré que les crises économico-financières se révèlent un terrain intéressant à étudier dans le cadre de l'analyse du discours et que l'analyse argumentative que nous avons développée à propos de ce débat peut s'appliquer à d'autres situations similaires et éclairer ainsi le mécanisme derrière les prises de décision. 


\section{BIBLIOGRAPHIE}

BENoIT W. L., «Image Repair Discourse and Crisis Communication », Public Relations Review, 23(3), 1997, p. 177-186.

CANCELli F., La retorica a Gaio Erennio, Milano, Mondadori, édition de 1992.

Ducrot O., Le dire et le dit, Paris, Minuit, 1984.

EEMEREN F. H. vAN et GRootendorst R., A Systematic Theory of Argumentation. The Pragma-Dialectical Approach, Cambridge, Cambridge University Press, 2004.

PALMIERI Rudi, « Regaining Trust through Argumentation in the Context of the Current Financialeconomic Crisis », Studies in Communication Science, 9(2), 2009, p. 59-78.

PALMIERI Rudi, « Text types, activity types and the genre system of financial communication ", 2011, dans L. Gautier (ed.), Les discours de la bourse et de la finance. Forum Für FachsprachenForschung, Berlin, Frank und Timme (sous presse).

RigotTi E., « Relevance of Context-bound Loci to Topical Potential in the Argumentation Stage », Argumentation, 2006, 20(4), p. 519-540.

RigotTI E., «Locus a causa finali », L'analisi Linguistica e Letteraria (special issue 2/2), 2008, p. 559-576.

RigotTi E., « Whether and how Classical Topics can be revived in the Contemporary Theory of Argumentation », dans F. H. van Eemeren et B. J. Garssen (éds), Pondering on Problems of Argumentation, New York, Springer, 2009, p. 157-178.

RigotTi E. et GrECo Morasso S., « Argumentation as an Object of Interest and as a Social and Cultural Resource ", dans N. Muller-Mirza et A. N. Perret-Clermont (éds), Argumentation and Education, New York, Springer, 2009a, p. 9-66.

RigotTI E. et GRECO MORASSO S., « Editorial \& Guest Editors' Introduction: Argumentative Processes and Communication Contexts ", Studies in Communication Sciences, 9(2), 2009b, p. 5-18.

RigotTI E. et PALMIERI R., « Analyzing and evaluating complex argumentation in an economicfinancial context ", dans C. Reed et C. W. Tindale (éds), Dialectics, Dialogue And Argumentation. An Examination Of Douglas Walton's Theories Of Reasoning, London, College, 2010, p. 85-99.

Rocci A., " Manoeuvring with Voices: The Polyphonic Framing of Argumentsin an Institutional Advertisement ", dans F. H. van Eemeren (éd.), Examining Argumentation in Context. Fifteen Studies on Strategic Manoeuvring, Amsterdam, John Benjamins, 2009, p. 257-283.

Toulmin S. E., The uses of argument, Cambridge, Cambridge University Press, 1958.

Ziegelmueller G. et Kay J., Argumentation : Inquiry and Advocacy, Boston, Allyn and Bacon, 1997.

\section{ANNEXES}




\section{Annexe 1. - Article-opinion de Rick Wagoner}

\section{THE WALL STREET JOURNAL.}

OPINION

NOVEMBER 19, 2008

Why GM Deserves Support

Short-term government backing can preserve a vital industry.

By RICK WAGONER

Much has been said about the impact of the credit crisis on U.S. auto makers, and whether or not the government should assist the industry during this extraordinary financial turmoil. In these discussions, many critics simply ignore the substantial changes that U.S. auto companies have already made - changes much like those the critics are calling for as part of any aid package.

At General Motors, we have been responding to fierce competition here and abroad by transforming our business. Over the past decade, we have taken tough actions to cut costs, at the same time investing billions in fuel-efficient vehicles and new generations of advanced propulsion technologies.

On the cost-cutting side, we have been streamlining our U.S. operations while simultaneously improving quality and productivity. Since 2000, we have reduced our U.S. hourly workforce by $52 \%$, from 133,000 to 64,000 , through buyouts and other programs. During the same period, we have cut our U.S. salaried employment from 44,000 to fewer than 30,000 , and reduced our U.S. executive ranks by $45 \%$.

However, we know we cannot just slash our way to prosperity. We have closed the quality and productivity gaps with the imports, as confirmed by J.D. Power and Associates (the consumer ratings firm) and the Harbour Report (which benchmarks North American plant-floor performance). New GM product programs launched earlier this decade have produced award-winning cars and crossovers like the Saturn Aura, Cadillac CTS and Buick Enclave. And that is just the beginning.

The new Chevy Malibu is a clear response to critics who say that GM cannot build cars that customers want. The Malibu leads its segment in highway fuel economy at $33 \mathrm{mpg}$ (2009 EPA figures), and was named best midsize car for initial quality in the most recent J.D. Power and Associates study. No surprise that, even in the worst car market in decades, Malibu sales are up $39 \%$ so far this year.

GM has also been working to re-establish its leadership in advanced propulsion technology. We have committed to producing the Chevy Volt - a revolutionary car that can go 40 miles on electricity alone - in 2010 in a U.S. factory. We are expanding our family of hybrid vehicles, investing in advanced biofuels, and continuing development of hydrogen fuel-cell vehicles. We are also applying our global technical resources to improve the fuel economy of conventional vehicles.

Just one example : The new Chevy Cruze, a global compact car that goes into production in Ohio in 2010, is expected to lead its segment in fuel economy. 
GM's commitment to these new products and technologies will help everyone. Consumers will benefit from lower fuel costs, our nation will use less imported petroleum, and our air will become cleaner. Development and production of these new vehicles here in the U.S. will maintain our nation's competitive standing and provide good paying jobs here at home. It also ensures that the U.S. does not trade its current dependence on imported oil for a future dependence on imported batteries and technology.

The auto industry may be historically anchored in Détroit, but it reaches into every state and community in our nation. Take Kansas, for example. GM assembles the Malibu and the Saturn Aura in Fairfax, Kan., making us a major employer there. The wages of our 2,500 workers flow into that community through spending on everything from mortgage payments to high-school bake sales.

Fairfax is not unusual. U.S.-based companies have 105 assembly and component plants in 20 states, including California, Texas, Louisiana and Maryland, states not typically thought of as auto country. GM, Ford and Chrysler last year purchased $\$ 156$ billion in parts, materials and services, supporting jobs in all 50 states.

Because of GM's deep commitment to its employees, dealers and communities, the company has been restructuring itself without the storm and drama some pundits mistake for actual progress. Working with the UAW, we have transformed our labor agreements to close the competitive gap. We have taken $\$ 9$ billion out of our annual structural costs since 2005 alone, and we have substantially reduced legacy costs inherited from decades past. In the face of the current credit crisis, we have moved to improve our liquidity by $\$ 20$ billion through 2009 through cuts in salaried employment, capital spending and other areas.

The future of the domestic auto business is critical to the health of the U.S. economy. It is a vital engine of economic growth and a foundation of economic stability. It remains a path of upward mobility for millions of American families. For America to compete in the global marketplace in the 21st century, it needs a strong manufacturing base and a vital domestic auto industry.

Nearly a half-century ago President Kennedy declared that his generation of Americans was living in extraordinary times and facing extraordinary challenges. Our times are no less challenging. They demand solutions that are creative and courageous.

Short-term government support to bridge the current financial crisis will enable GM to continue as an engine for prosperity and as a creator of vehicles and technologies that America needs. Such assistance will save millions of jobs now, and produce enormous benefits for years to come.

Mr. Wagoner is chairman and CEO of General Motors Corp.

\section{Annexe 2. - Article-opinion de Paul Ingrassia}

\section{THE WALL STREET JOURNAL. \\ WSJ.com}

The Auto Makers Are Already Bankrupt

Admitting the obvious is their best chance to restructure.

OPINION 
NOVEMBER 21, 2008

By PAUL INGRASSIA

The moment of truth in the nation's automotive bailout debate might have come this week. As the CEOs of GM, Ford and Chrysler begged Congress for federal aid, a Detroit radio talk-show host asked whether Michigan, as well as the car companies, should get assistance. The state is being hit by an economic hurricane, he said, just as New Orleans was hit by a natural hurricane.

Huh? Will the victimology myth never end? Hurricane Katrina was an act of God. The car crisis is an act of man. For the difference, consult the Bible. Any version will do.

Yesterday, congressional leaders gave the car companies until Dec. 2 to come up with viable business plans and renew their request for aid. Meanwhile, it's worth examining the myths that are shaping this debate. One is GM's assertion that "bankruptcy is not an option". In truth, GM already has conceded that it's bankrupt - by publicly stating it's nearly out of cash and needs emergency assistance. The company hasn't made a formal bankruptcy filing, which is no small matter. But it has declared bankruptcy everywhere else. Chrysler, at this week's Senate committee hearing, did the same.

A second myth is that management changes in Detroit would be pointless. GM CEO Rick Wagoner said he wouldn't resign to secure federal aid for his company. This was like Louis XIV saying, “L'État c'est moi”. Mr. Wagoner explained that he didn't see "what purpose would be served". Well, the same one served by the presidential election in this country three weeks ago : to bring in somebody new to try some fresh ideas to fix things.

Mr. Wagoner has been GM's chief executive officer for eight years. Even before this year's calamity struck (the company lost $\$ 181,000$ per minute in the second quarter), the company's U.S. market share, financial results and stock price had plunged precipitously.

At Chrysler, CEO Robert Nardelli has been on the scene just a year. Before that he was at Home Depot, where he took a \$ 210 million departure package when the board wanted him out. There's no reason to begrudge Mr. Nardelli that money. But any plan to save Chrysler will inflict great hardship on dealers, suppliers, workers and managers - and even if Mr. Nardelli is a great executive talent, he isn't the guy to lead the clarion call for sacrifice, despite his recent offer to work for \$ 1 a year. Symbols are important here, which is why the spectacle of the Detroit CEOs swooping into Washington on corporate jets to ask for money was so jarring.

Ford CEO Alan Mulally has been on the job just over two years. He seems to be making the right moves - cutting costs, eliminating the dividend early on, revamping product plans, mortgaging assets to raise money to fund the turnaround, etc. That's why Ford, while not in great shape, is in a materially better position than the other two.

Mr. Mulally is the Detroit chief executive I'd keep on the job. But that still doesn't mean it's right to hand federal aid to Ford or any of the other companies without requiring a bankruptcy restructuring in return.

Which raises the third myth: Bankruptcy means death. In fact, it means getting a second chance. Detroit's car companies point, correctly, to the cost cuts, labor concessions and other stringent measures that they've enacted in recent years.

Ron Gettelfinger, the president of the United Auto Workers union, got his members to accept two-tier wages and big concessions on the health-care and retirement plans. 
Nonetheless, far too many valid contractual claims remain on the car companies' revenue streams from dealers, employees, retirees and others for these companies to survive - even if we get a modest economic recovery soon. The companies remain saddled with cumbersome contracts with the UAW that make work rules and plant procedures a constant challenge. A bankruptcy trustee or receiver could cut through all this quickly and give the companies a fresh start.

Myth number four is that banning executive bonuses or requiring more fuel-efficient cars will save Detroit, and are strings that should come with any federal aid. Executive pay isn't the problem in Detroit; and the companies will have to build more fuel-efficient cars to satisfy the market, not to meet mandates. These would be pseudo-strings designed to appease organized labor and the environmental lobby. Instead of saving Detroit, they'll pave the way for a bigger bailout later on.

Finally, the fifth myth is that a merger of GM and Chrysler will propel both companies to prosperity. Some of the slide-shows making the rounds on Wall Street assume that a merged company would have a $30 \%$ market share, slightly less than the two companies now have combined. It isn't true. The elimination of duplicate brands, models and dealers would push a combined market share down to $25 \%$ or less. The revenue projections behind a potential merger seem greatly inflated. GM has massive problems of its own to address without taking on those of Chrysler, which needs a profitable, and committed, foreign buyer.

The biggest beneficiaries of a GM-Chrysler merger would be Cerberus, the private-equity firm that owns Chrysler, and the big banks that hold billions of Chrysler bonds that they haven't been able to sell. The bonds were used in Cerberus's purchase of Chrysler from Daimler. The banks expected to sell the bonds to investors, but have been left holding billions in Chrysler debt that they'd dearly love to unload.

\section{Annexe 3. - Argumentations des Big Three contraires à l'hypothèse « erreur humain »}

Mr. Chairman, members of the Committee, I appreciate this opportunity to address the current economic and financial crisis, the impact it is having on the automotive industry, and the need for immediate action. (R. Nardelli, 18 novembre 2008, source : <http:// autoinsane.com>)

We are asking for assistance for one reason: to address the devastating automotive industry recession caused by our nations' financial meltdown, and the current lack of consumer credit, which has resulted in the critical lack of liquidity within our industry. ( ibid.)

This unprecedented pressure on our industry, which is the result of a financial crisis that was not of our industry's making, is coming just at the time when our efforts to restructure Ford have finally begun to bear fruit. (A. Mulally, 18 novembre 2008, source : $<$ http://media.ford.com>)

We are in an economic situation now, with the credit crisis and the financial and the banking issues, that we really, more than ever, the automobile industry needs to be part of the solution. (ibid.) 
What exposes us to failure now is not our product lineup, or our business plan, or our long-term strategy. What exposes us to failure now is the global financial crisis, which has severely restricted credit availability, and reduced industry sales to the lowest per-capita level since World War II. (R. Wagoner, 18 novembre 2008, source : <http://s.wsj.net>)

Such a level of economic devastation would far exceed the government support that our industry needs to weather the current crisis. That's why this is about much more than “just” Detroit... it's about saving the U.S. economy from a catastrophic collapse. (ibid.)

\section{NOTES}

1. Ce n'est pas par hasard si les sciences économiques sont très souvent interprétées comme des sciences dures et non des sciences humaines et sociales. Ce préjugé est sans doute renforcé par une forte mathématisation de la finance, qui amène à croire que les interactions financières sont déterminées par des lois nécessaires qui excluent la liberté et la décision de l'être humain responsable.

2. «Stock issues, very simply, are hunting grounds for arguments. They provide general phrasing of potential issues that correspond to the inherent obligations of the advocate of change. » (Ziegelmueller et Kay, 1997)

3. Une argumentation pratiquement identique est utilisée par le directeur de Ford quand il répond à une question posée par un journaliste :

John Roberts (CNN) : Why should taxpayers give you any of their hard-earned money?

Alan Mulally (Ford) : Well, I think the compelling argument is that the automobile industry is just absolutely essential to the United States' economy. We are in an economic situation now, with the credit crisis and the financial and the banking issues, that we really, more than ever, the automobile industry needs to be part of the solution. And the only thing that we're asking for is to set up a bridge loan mechanism so that if the economy continues to deteriorate in the near term, that we could access that so we could continue to invest in the products that people really do want and value and help be part of this economic recovery.

(Source: <http://articles.cnn.com/2008-11-18/us/mulally.automaker.bailout_1_alan-mulallyfuel-efficiency-ford-ceo?_s=PM:US>)

4. Parmi les articles de presse qui ont avancé une opinion contraire au bailout, mentionnons : Paul Ingrassia, How Détroit Drove Into a Ditch, WSJ, 25 octobre 2008 ; David Yermack, Just Say No to Détroit, WSJ, 15 novembre 2008, Michael Levine, Why Bankruptcy Is the Best Option for GM, WSJ, 17 novembre 2008 ; Paul Ingrassia, The Auto Makers Are Already Bankrupt, WSJ, 21 novembre 2008.

\section{RÉSUMÉS}

La crise financière qui s'est déclenchée en 2007 a fortement affecté l'industrie automobile, en aggravant une situation déjà difficile pour les entreprises, en particulier « les Trois Grands » (The Big Three) de Détroit (Ford, General Motors, Chrysler). Cette crise dans la crise, s'il faut en croire les arguments des entreprises, était étroitement liée au resserrement du crédit.

À l'opposé, de nombreuses critiques ont été formulées contre les trois entreprises, accusées de 
n'avoir pas été capables de s'adapter aux développements du secteur. Par conséquence, la question des prêts de l'État sollicités par les Big Three a provoqué un débat acharné où différentes interprétations concernant la crise financière et l'industrie automobile ont été avancées : crise financière comme seule cause des ennuis du secteur automobile ou crise financière portant le coup de grâce à un secteur déjà en crise?

Cette analyse se focalise sur la fonction de ces interprétations dans les structures inférentielles des arguments qui ont cadré ce débat. Plus précisément, chaque interprétation représente une valeur ou principe partagé - Endoxon dans la théorie de l'argumentation - qui permet de fonder le parcours argumentatif désiré. Une attention particulière est apportée à la caractérisation de la crise, dont l'interprétation comme événement presque naturel ("acte de Dieu ») ou comme résultat d'une action («erreur humaine ») se révèle fondamentale par rapport à la politique financière à suivre. L'analyse porte sur des articles d'opinion publiés par plusieurs médias (comme le Wall Street Journal, BBC News) entre la fin de 2008 et le début de 2009, et sur les documents principaux qui ont servi aux directeurs d'entreprise dans l'argumentation de leur position.

The 2007 financial crisis significantly affected the automobile industry, particularly the Big Three of Detroit (Ford, General Motors, and Chrysler). According to management, theirs was a "crisis within the crisis" linked to the credit crunch. On the other hand, critics charged the Big Three had failed to adapt to new industrial developments. Consequently, federal government support to these companies generated debate about whether the financial crisis was a specific problem experienced by the automobile sector or the coup de grace administered to an already troubled industry.

This analysis focuses on the inferential configurations of the main arguments which characterized the Detroit debate. More precisely, the opposite ways in which the financial crisis is interpreted constitute different shared values or principles - called Endoxon in argumentation theory - which ground the desired argumentative path. Particular attention is paid to the strategies through which the crisis is framed as a substantially natural event ("Act of God") or as the outcome of human action ("Act of Man"), a decisive factor with regard to obtaining the financial aid claimed.

The analysis is based on news stories and comments in different media (e.g. Wall Street Journal, $B B C$ News) late 2008 and early 2009, and documents on the basis of which the management of Ford, Chrysler and GM argued their position.

\section{INDEX}

Mots-clés : action, argumentation, Argumentum Model of Topics, communication financière, crise, Endoxon, industrie automobile de Détroit, maxime, mérite, responsabilité

Keywords : Act of God, Act of Man, Argumentum Model of Topics, Detroit automobile industry, Endoxon, financial communication, financial crisis, managerial argumentation

\section{AUTEUR}

\section{RUDI PALMIERI}

Post-doc researcher. Institute of Argumentation, Linguistics and Semiotics, USI, University of Lugano, Switzerland 